\title{
Power scaling of a hybrid microstructured Yb-doped fiber amplifier
}

\author{
Cody Mart ${ }^{1,2}$, Benjamin Pulford ${ }^{1}$, Benjamin Ward $^{3}$, Iyad Dajani ${ }^{1}$, Thomas Ehrenreich ${ }^{1}$, Brian Anderson ${ }^{1}$, \\ Khanh $\mathrm{Kieu}^{2}$, and Tony Sanchez ${ }^{1}$ \\ ${ }^{1}$ U.S. Air Force Research Laboratory, 3550 Aberdeen Ave SE, Kirtland AFB, New Mexico 87117 USA \\ ${ }^{2}$ University of Arizona, College of Optical Sciences, 1630 E University Blvd, Tucson, Arizona 85721 USA \\ ${ }^{3}$ Department of Physics, U.S. Air Force Academy, HQ USAFA/DFP 2354 Fairchild Dr., USAF Academy, \\ Colorado 80840 USA
}

\begin{abstract}
Hybrid microstructured fibers, utilizing both air holes and high index cladding structures, provide important advantages over conventional fiber including robust fundamental mode operation with large core diameters $(>30 \mu \mathrm{m})$ and spectral filtering (i.e. amplified spontaneous emission and Raman suppression). This work investigates the capabilities of a hybrid fiber designed to suppress stimulated Brillouin scattering (SBS) and modal instability (MI) by characterizing these effects in a counter-pumped amplifier configuration as well as interrogating SBS using a pump-probe Brillouin gain spectrum (BGS) diagnostic suite. The fiber has a $35 \mu \mathrm{m}$ annularly gain tailored core, the center doped with $\mathrm{Yb}$ and the second annulus comprised of un-doped fused silica, designed to optimize gain in the fundamental mode while limiting gain to higher order modes. A narrow-linewidth seed was amplified to an MI-limited $820 \mathrm{~W}$, with near-diffraction-limited beam quality, an effective linewidth $\sim 1 \mathrm{GHz}$, and a pump conversion efficiency of $78 \%$. Via a BGS pump-probe measurement system a high resolution spectra and corresponding gain coefficient were obtained. The primary gain peak, corresponding to the $\mathrm{Yb}$ doped region of the core, occurred at $15.9 \mathrm{GHz}$ and had a gain coefficient of $1.92 \times 10^{-11} \mathrm{~m} / \mathrm{W}$. A much weaker BGS response, due to the pure silica annulus, occurred at $16.3 \mathrm{GHz}$. This result demonstrates the feasibility of power scaling hybrid microstructured fiber amplifiers
\end{abstract}

Keyword: Photonic Bandgap Fiber, Photonic Crystal Fiber, Mode Instability, Stimulated Brillouin Scattering

\subsection{INTRODUCTION}

Fiber waveguides relying on both air holes and high-index rods in the cladding to achieve wave guidance, herein referred to as Hybrid fibers, possess numerous advantages for power scaling in diode-pumped, Yb-doped fiber amplifier architectures. With suitable waveguide designs, mode area scaling can be realized with robustly single-mode fibers having cores $>30 \mu \mathrm{m}$ in diameter [1-2]. Larger cores greatly reduce intensity-dependent nonlinear optical interactions such as stimulated Brillouin scattering (SBS) and stimulated Raman scattering (SRS). Spectral filtering via the photonic bandgap (PBG) effect can be implemented to suppress unwanted amplified spontaneous emission (ASE), Raman, and other nonlinear processes. Confinement losses to the fundamental core mode are not as caustic as in all-solid PBG fibers because modified total-internal reflection via the air holes offers more robust confinement. Additionally, the single-mode spectral region is broader than for all-solid PBGF [1].

Power scaling of Yb-doped Hybrid fibers has attracted attention for amplifying wavelengths $>1120 \mathrm{~nm}$ [1-3]. The advantage of utilizing Hybrid fiber in this spectral domain is that mitigation of parasitic lasing in the strong ytterbium gain region of 1030-1100 $\mathrm{nm}$ is possible. The $1178 \mathrm{~nm}$ laser source developed by Peterson et al demonstrated the ability of these fibers to reach longer wavelengths but was much too broad in linewidth to demonstrate the ability of the photonic bandgap structure to reduce SBS [2]. Interrogating this property is essential for power scaling of beamcombinable Hybrid fiber amplifiers.

In addition to SBS, a major power-limiting factor of large mode area (LMA), narrow-linewidth Yb-doped amplifiers is the rapid onset of modal instability (MI), a time-dependent coupling of energy between the fundamental mode and higher-order modes within the fiber core [4]. The goal of this research is to interrogate SBS and MI behavior in a LMA

Fiber Lasers XIV: Technology and Systems, edited by Craig A. Robin, Ingmar Hartl, Proc. of SPIE Vol. 10083, 100830X · @ 2017 SPIE · CCC code: 0277-786X/17/\$18 - doi: 10.1117/12.2249863 
Yb-doped Hybrid fiber amplifier. A Pump/Probe Brillouin gain spectrum diagnostic suite is used to interrogate SBS, and the MI limits are tested in a free-space counter-pump amplifier.

Annular gain tailoring of the fiber core is employed to limit MI in this work [5]. In this scheme, the Yb-doped region of the core is spatially segregated to overlap strongly with the fundamental core mode while overlapping weakly with higher-order modes. Pure silica is used in the outer annulus of the core with strong higher-order mode overlap.

Mode area scaling is implemented to mitigate SBS. Since the gain tailored core is segmented into active and passive regions, each with a different acoustic response, one might assume that having multiple acoustic frequencies in the Brillouin gain spectrum would increase SBS threshold. It will be shown, however, that the fundamental mode overlap with the active $\mathrm{Yb}$ region dominates the SBS gain dynamics and that SBS is not reduced from gain tailoring.

\subsection{FIBER SPECIFICATIONS}

The SBS and MI behavior of the hybrid fiber shown in Figure 1, developed in collaboration with NKT Photonics, is investigated under this effort. The core and inner-cladding diameters are $35 \mu \mathrm{m}$ and $350 \mu \mathrm{m}$ respectively. Gain tailoring of the core is implemented in an annular ring design whereby a pure silica ring surrounds an active Yb-doped central region. The center region is co-doped with $\mathrm{Al}$ and $\mathrm{F}$ to match the optical refractive index of $\mathrm{Yb}$-doped region with that of pure silica, creating an optically uniform core. Figure 1 shows a schematic of the fiber design to the left and the right shows a microscope image of the fiber facet. The high-index germanium-doped cladding structures responsible for photonic bandgap guidance are positioned in three offset rows of 3 high index structures per column. The hexagonal air hole pattern responsible for modified TIR guidance measures $115 \mu \mathrm{m}$ flat-to-flat and $130 \mu \mathrm{m}$ tip-to-tip. The pump NA is $0.5-0.6$ and the attenuation at the pump wavelength is estimated at $1.6 \mathrm{~dB} / \mathrm{m}$. The second clad diameter is $590 \mu \mathrm{m}$ and has flat coil control surfaces in the same axis as the high-index inclusions to define the bend axis.
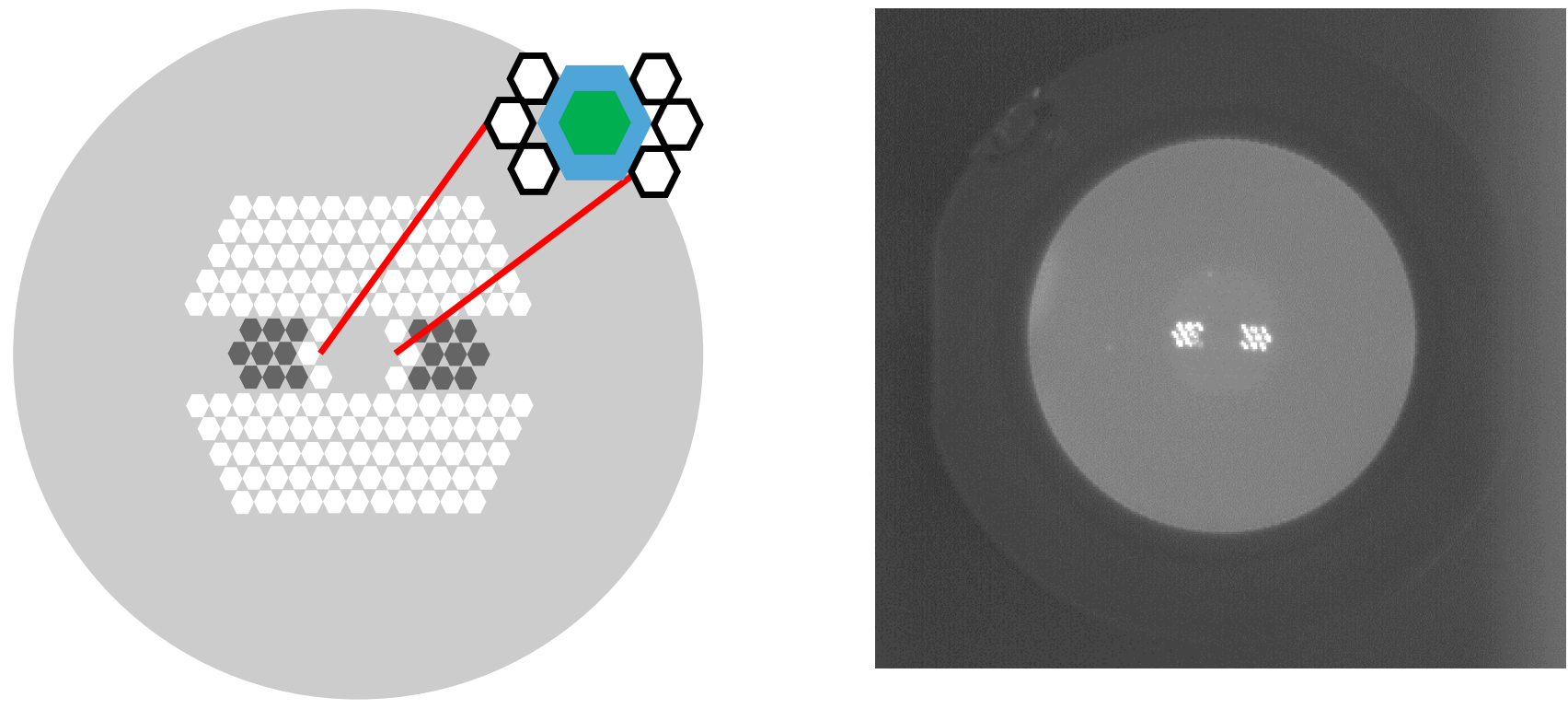

Figure 1

A schematic of the fiber design is shown on the left. The inset shows the gain tailored core with a ring of pure silica (blue) surrounding the $\mathrm{Yb}$-active (green) region. A microscope image of the fiber is shown to the right.

\subsection{BRILLOUIN GAIN SPECTROSCOPY}

A dual-source Pump Probe Brillouin gain spectrum (BGS) diagnostic suite was utilized to measure the BGS and to estimate the Brillouin gain coefficient, $\mathrm{g}_{\mathrm{B}}$. The Pump Probe measurement simulates amplification of the Stokes light via the Brillouin scattering process by engendering a longitudinal optical interference pattern from independent, counterpropagating, frequency detuned oscillators coupled to opposing ends of the fiber. The optical interference pattern stimulates the electrostrictive process that generates the traveling wave Bragg structure that scatter the higher-frequency 
pump into a lower-frequency probe mode. The BGS can be measured by recording the power coupling as a function of frequency-detuning with the peak interaction occurring at the Brillouin shift.

A schematic of the dual-source Pump Probe measurement system is shown in Figure 2. Two non-planar ring oscillators (NPROs) are separately amplified and free-space coupled to opposing ends of the Hybrid fiber. Glass wedges are used to directionally separate the pump from the probe so that the monitoring of the power dynamics with near-polarizationinsensitivity can be realized. Frequency-detuning is achieved via temperature-tuning of the probe NPRO crystal. The pump is amplified to $\sim 40 \mathrm{~W}$ in a single-frequency $\mathrm{Yb}$-doped amplifier from IPG Photonics; which maintains a PER $>20$ $\mathrm{dB}$ and has a near diffraction limited beam quality $\left(\mathrm{M}^{2}<1.2\right)$. A polarization scrambler is used to scramble the probe so that interaction with the low-PER pump can be captured with all polarization states available. The non-PM fiber under tests degrades the pump polarization to a PER $<13 \mathrm{~dB}$. The probe is amplified in a non-PM Yb-doped amplifier which emits an effectively randomly polarized, diffraction-limited beam with PER $<0.5 \mathrm{~dB}$. The frequency difference between the pump and probe beams is measured on a heterodyne system. To ensure that the diagnostic captured only interactions between counter-propagating fundamental core modes, a spatial beam analyzer was used to estimate $\mathrm{M}^{2}$ based on a $1 / \mathrm{e}^{2}$ hyperbolic fit. Both the pump and probe beams were coupled to the test fiber with a near-diffraction-limited $\mathrm{M}^{2}<1.2$.

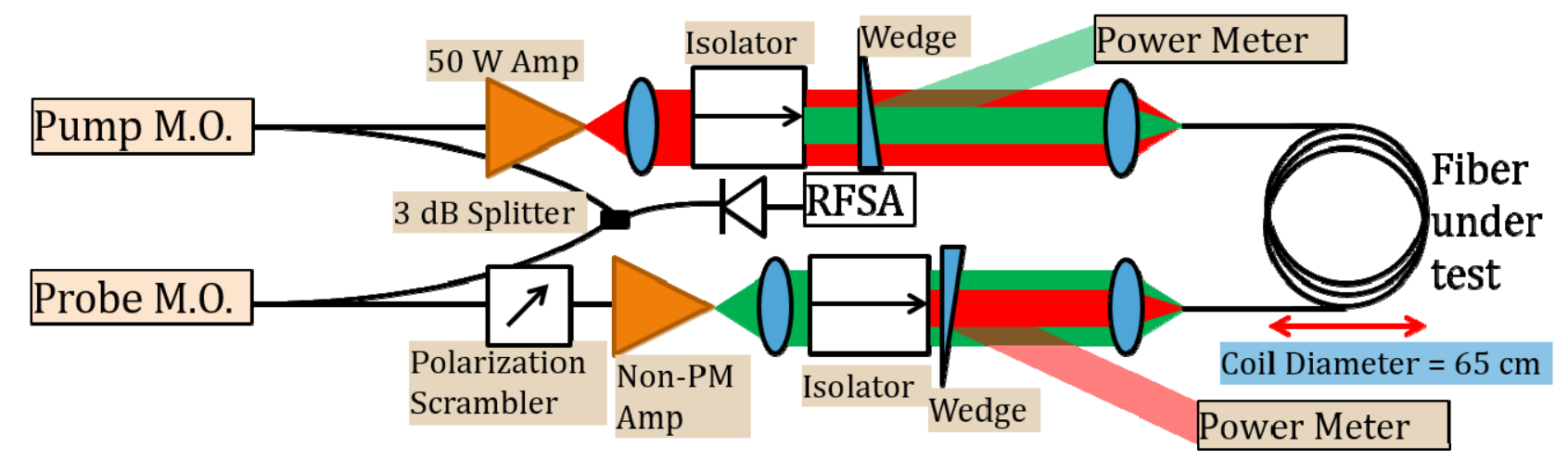

Figure 2

The Pump Probe Brillouin gain spectrum diagnostic is shown above. M.O. = master oscillator, RFSA = radio frequency spectrum analyzer,

Two BG spectra are shown in Figure 3. The long-range scan on the left shows two distinct responses at $15.9 \mathrm{GHz}$ and 16.3 GHz. The $15.9 \mathrm{GHz}$ feature, which has a measured full-width at half-maximum (FWHM) bandwidth of $42 \mathrm{MHz}$, corresponds to the response of the $\mathrm{Yb}$-doped center region of the core and the $16.3 \mathrm{GHz}$ feature corresponds to the response from the passive fused silica annulus. To excite a resolvable response from the passive silica, pump powers sufficient to induce pump depletion at the Yb-doped response were needed. Since $\mathrm{Yb}$ lowers the BG coefficient in pure silica, it can be inferred that the fundamental core mode overlaps poorly with the pure silica annulus.

The small-signal BG coefficient from the dominant $\mathrm{Yb}$-doped response was estimated by simulating the $2 \times 2$ coupled differential equations describing power coupling via the Brillouin scattering process [6], with $\mathrm{g}_{\mathrm{B}}$ being tuned to match the simulated power with the power coupling observed in experiment. Since a polarization scrambler was used to depolarize the probe, the observed $\mathrm{g}_{\mathrm{B}}$ can be decomposed into an average of the most responsive polarization and least responsive polarization. To obtain the natural $g_{B}$, the observed $g_{B}$ is multiplied by two [7]. The right plot in Figure 3 shows the BGS due to the $\mathrm{Yb}$ region scaled to the estimated $\mathrm{g}_{\mathrm{B}}$. 


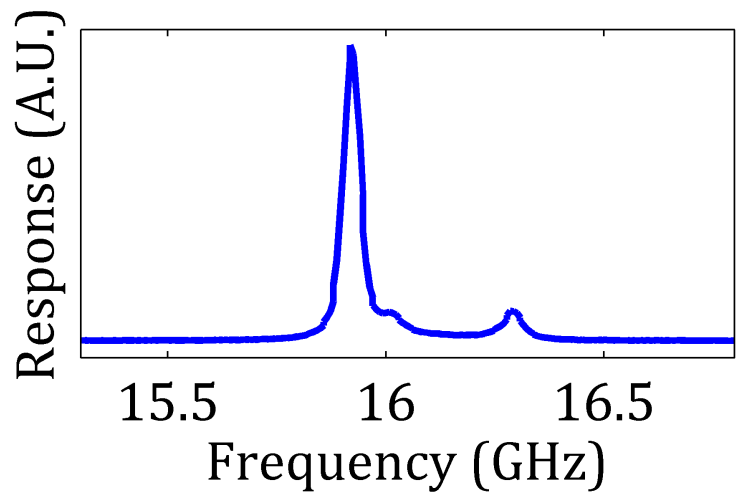

Figure 3

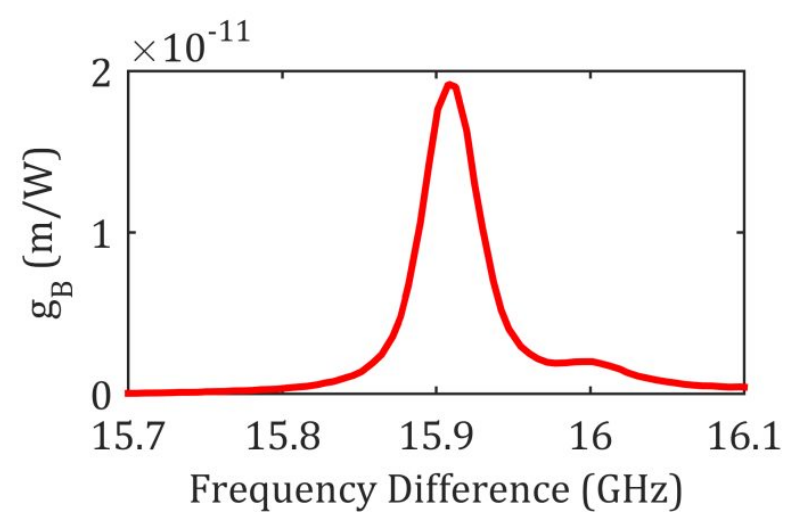

Frequency Difference (GHz)

The BG spectra above show the wide scan BGS (left) and the BGS scaled the $g_{B}$ peak due to the Yb-doped core segment (right)

The natural BG coefficient of $1.92 \times 10^{-11} \mathrm{~m} / \mathrm{W}$ is too high for single-frequency power scaling. Although not ideal for characterizing SBS in a fiber amplifier, the threshold equation of $21\left(\alpha A_{e f f} / g_{B}\right)$ [8] provides a critical power of $25 \mathrm{~W}$ with a $35 \mu \mathrm{m}$ mode field diameter and $0.1 \mathrm{~dB} / \mathrm{m}$ loss coefficient. To mitigate SBS, a pseudo-random bit sequence (PRBS) phase modulation scheme was employed to frequency-broaden the final amplifier. A 15 bit pattern was applied over a $1 \mathrm{GHz}$ envelope with RF filtering implemented to reduce the spectrum to the first envelope in the sinc-squared pattern. The resulting tones of the phase modulator were spaced by $66.6 \mathrm{MHz}$; which is $\sim 1.5 \mathrm{x}$ greater than the BG FWHM of the primary response. Zeringue et al argue in [9] that optimal SBS suppression for a given phase-broadened bandwidth is when the spacing of the phase modulator tones are 2-3 times greater than the BG FWHM. The bit pattern in this study is not optimal, but it will be shown that the PRBS scheme sufficiently suppressed SBS.

\subsection{POWER SCALING}

To demonstrate power scaling with the hybrid fiber, the free space coupled experimental setup in Figure 4 was used. The counter propagating pump and seed signals were coupled to opposing ends of the fiber. The $1064 \mathrm{~nm}$ NPRO seed signal is phase modulated using a magnesium-doped $\mathrm{LiNbO}_{3}$ crystal from EOspace; after which the seed signal is amplified to $\sim 30 \mathrm{~W}$ in a 3-stage amplifier, from IPG photonics, before being free-space coupled to the fiber core. The fiber has a $\sim 4$ degree angle cleave at both ends and is held in position on water-cooled chucks atop a 5-axis stage for free-space coupling. Dichroics are used to separate the $976 \mathrm{~nm}$ pump and $1064 \mathrm{~nm}$ signal. The fiber is coiled on a $65 \mathrm{~cm}$ diameter aluminum spool that is not actively cooled.

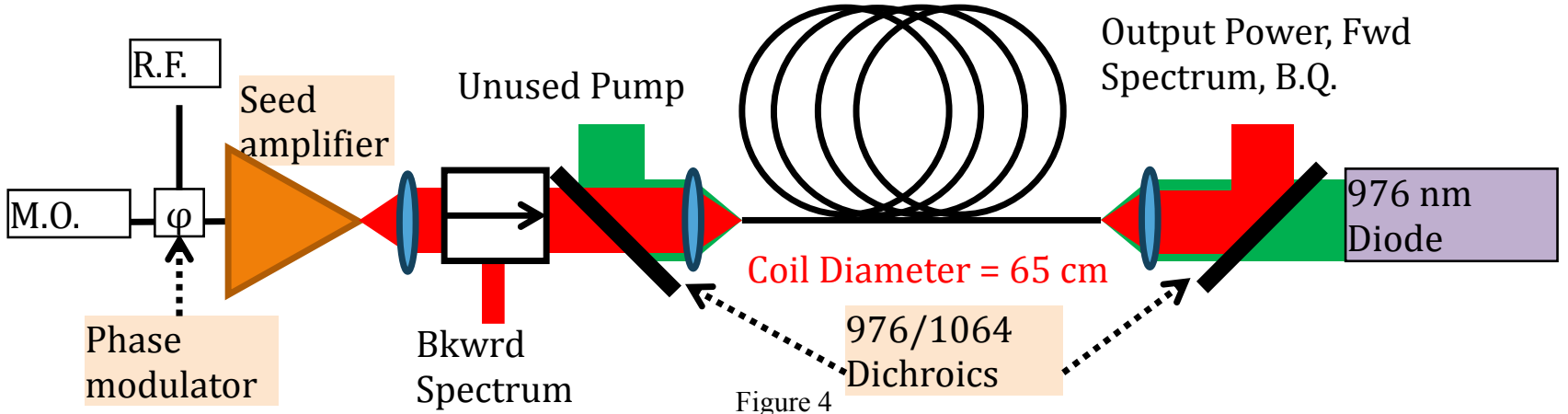

The experimental setup for the counter-pumped amplifier is shown above. M.O. = master oscillator, R.F. $=$ radio-frequency synthesizer

The diagnostics used to characterize amplifier performance include: power meters to measure the amplified signal power and unused pump power, optical spectrum analyzers (OSAs) to characterize the forward and backward signals from the amplifier, a spatial mode scanning $\mathrm{M}^{2}$ beam quality analysis machine, and a high-speed camera to capture the onset of MI. 
A $9 \mathrm{~m}$ length of Hybrid fiber was integrated into the setup shown in Figure 4 and power scaled. A MI-limited, $820 \mathrm{~W}$, near-diffraction-limited SBS-free output with $\sim 1 \mathrm{GHz}$ linewidth was achieved. A plot of the signal power versus total launched pump power is shown in Figure 5. The fiber demonstrated a total optical-to-optical efficiency of 78\%. Factors that reduced efficiency that are not present in standard step-index fiber includes the non-zero fundamental mode confinement loss in the Hybrid structure and the reduced overlap of the core mode with the Yb-doped region due to gain tailoring. Additionally, pump guidance in the high-index cladding structures is expected to affect conversion efficiency. The shaded region in the figure indicates signal power levels that were affected by MI.

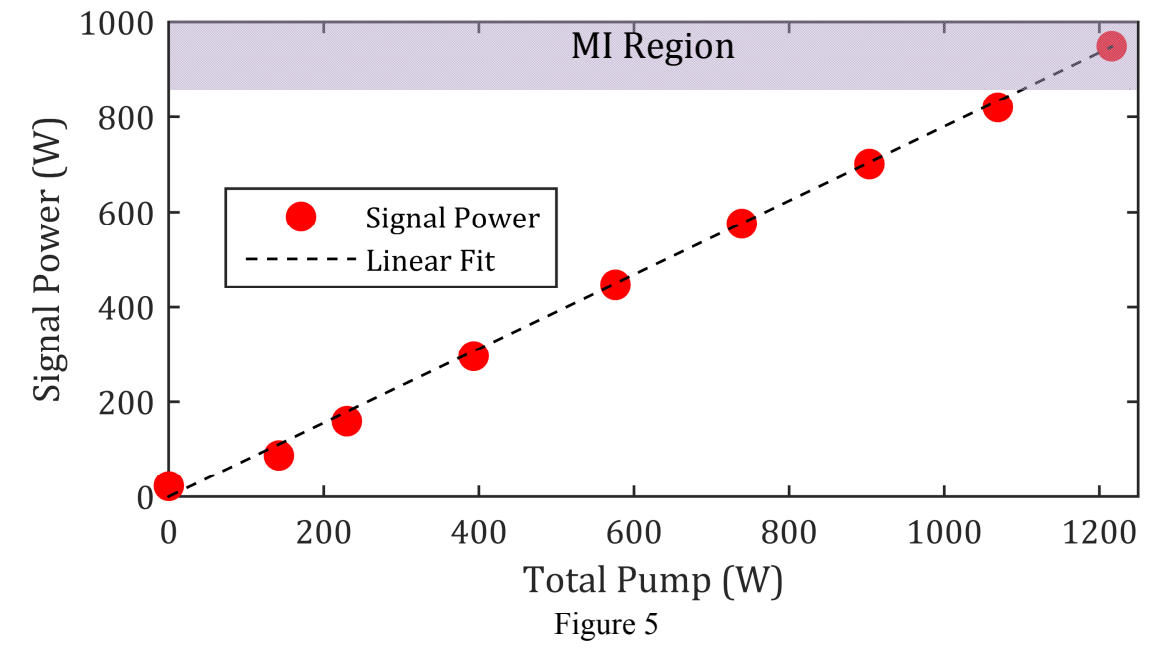

The output power vs total pump power plot is shown above along with a linear fit. The shaded region indicates that modal instability will be present.

A high-resolution OSA was used to separate the backward-propagating light into the Stokes and Rayleigh components. Figure 6 shows the zoomed-in OSA trace acquired at the highest recorded MI free power. No evidence of SBS is observed. The $1 \mathrm{GHz}, 15$ bit PRBS phase broadening scheme was very effective at suppressing SBS at these power levels.

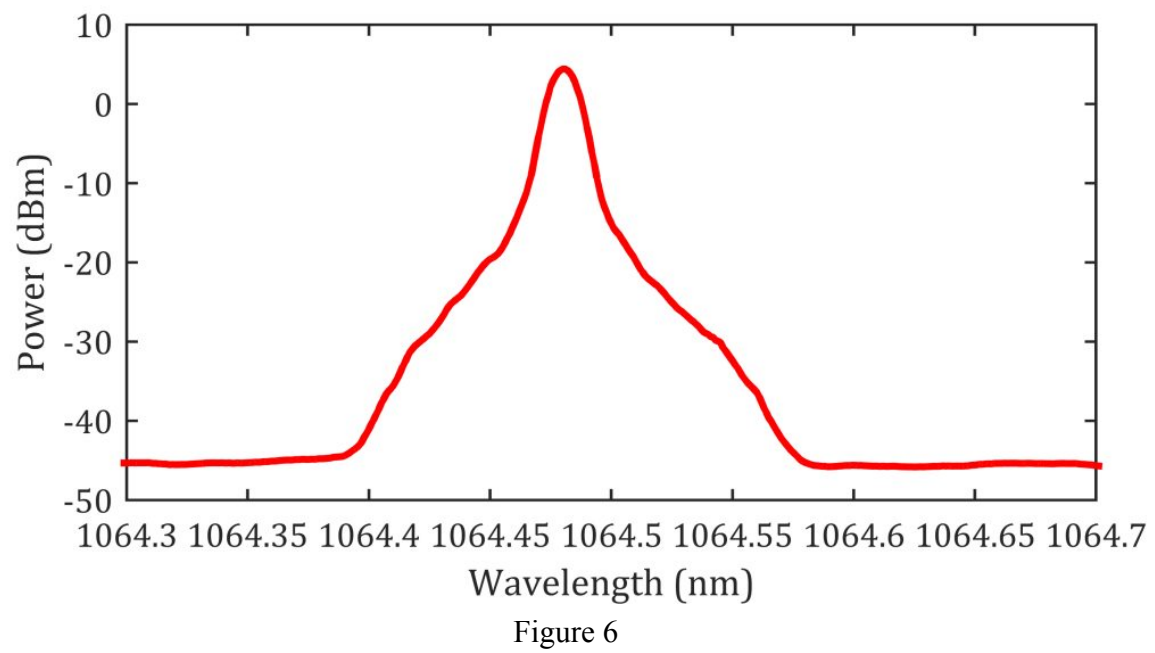

The backward optical spectrum is shown above. No evidence of Stokes light spaced $\sim 0.05 \mathrm{~nm}$ from the Rayleigh is resolved. 
As the power was scaled beyond $820 \mathrm{~W}$, dynamic mode variations were observed and beam quality was degraded; which indicated that the MI threshold had been surpassed. Figure 7 shows the average $\mathrm{M}^{2}\left(\left(\mathrm{M}_{\mathrm{x}}^{2}+\mathrm{M}_{\mathrm{y}}^{2}\right) / 2\right)$ versus signal power along with $\mathrm{M}^{2}$ measured in the plane of the high-index microstructures (x-axis) and air holes (y-axis) respectively. Neardiffraction-limited beam quality was maintained until $820 \mathrm{~W}$. The output power did not deviate from the $78 \%$ efficiency demonstrated in the MI-free regime; which indicates that confinement losses to the higher-order modes while the amplifier was experiencing MI were not significant.

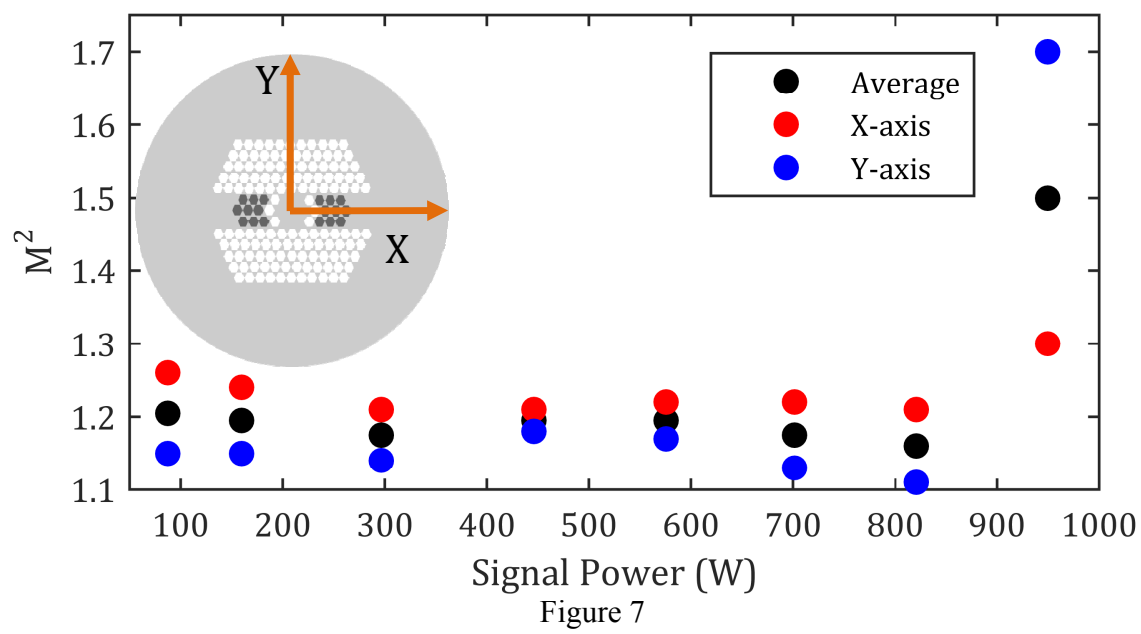

A plot of $\mathrm{M}^{2}$ versus signal power is shown above. The rapid increase in beam quality above $820 \mathrm{~W}$ is a result of MI onset. Inset, a schematic of the fiber showing the orientation of the air holes and high-index microstructures during measurement.

\subsection{CONCLUSIONS}

A Yb-doped Hybrid microstructured fiber was power scaled in a counter-pumped architecture to an MI-limited $820 \mathrm{~W}$, with beam quality near the diffraction limit and a phase broadened linewidth of $1 \mathrm{GHz}$. Our results indicate that Hybrid fibers are suitable candidates for integration into high power amplifiers in the $<1070 \mathrm{~nm}$ spectral regime of strong ytterbium gain.

A Pump/Probe Brillouin gain diagnostic estimated the BG coefficient at $1.92 \times 10^{-11} \mathrm{~m} / \mathrm{W}$; which was unsuitable for single-frequency power-scaling, and motivated the integration of a pseudo-random bit sequence phase broadening scheme. Gain tailoring of the optical gain was employed to mitigate MI. Overlap of the fundamental mode with the Ybdoped region is efficient from the BGS data. The observed total pump to signal efficiency of $78 \%$ from a $9 \mathrm{~m}$ fiber is on par with conventional Yb-doped fiber MOPA configurations and demonstrates that fundamental core mode confinement losses and gain tailoring do not strongly influence amplifier efficiency.

This demonstrates, to the best of our knowledge, the highest CW amplified power from an Yb-doped Hybrid fiber amplifier with a narrow linewidth. The non-observation of Stokes light in the amplifier indicates that the frequency broadening bandwidth can be reduced for the same operating power. Segmented acoustic tailoring and stress/temperature gradients can be applied if single-frequency power scaling is desired. The MI onset at $820 \mathrm{~W}$ indicates that further MI mitigation techniques must be implemented to achieve further power scaling.

\subsection{References}

[1] Peterson, S. R., Alkeskjold, T. T., Poli, F., Coscelli, E., Jørgensen M. M., Laurila, M., Lægsgaard, J., and Broeng, J., "Hybrid Ytterbium-doped large mode area photonic crystal fiber amplifier for long wavelengths," Opt. Exp. 20(6) 60106020 (2012). 
[2] Peterson, S. R., Chen, M., Shirakawa, A., Olausson, C. B., Alkeskjold, T. T., and Lægsgaard, J., "Large-mode-area hybrid photonic crystal amplifier at $1178 \mathrm{~nm}$," Opt. Lett. 40(8) 1741-1744 (2015).

[3] Goto, R., Mägi E. C., and Jackson, S. D., "Narrow-linewidth, $\mathrm{Yb}^{3+}$-doped, hybrid microstructured fibre laser operating at $1178 \mathrm{~nm}$," Elect. Lett. 45(17) 877-878 (2009).

[4] Eidam, T., Wirth, C., Jaugegui, C., Stutzki, F., Jansen, F., Hans-Jürgen, O., Schmidt, O., Schreiber, T., Limpert, J., and Tünnermann, A., "Experimental observations of the threshold-like onset of mode instabilities in high power fiber amplifiers," Opt. Exp. 19(14) 13218-13224 (2011)

[5] Robin, C., Dajani, I., and Pulford, B., "Modal instability-suppressing, single-frequency photonic crystal fiber amplifier with 811 W output power," Opt. Lett. 39(3) 666-669 (2014)

[6] Agrawal, G. P., [Nonlinear Fiber Optics, $5^{\text {th }}$ Ed.], Academic Press, Waltham, MA, 358 (2012).

[7] Horiguchi, T., Tateda, M., Shibata, N., and Azuma, Y., "Brillouin gain variation due to a polarization-state change of the pump or Stokes fields in standard single-mode fibers," Opt. Lett. 14(6) 329-331 (1989)

[8] Smith, R. G., "Optical power handling capacity of low-loss optical fibers as determined by stimulated Raman and Brillouin scattering," App. Opt. 11(11) 2489-2494 1972

[9] Zeringue, C., Dajani, I., Naderi, S., Moore, G. T., and Robin, C., "A theoretical study of transient stimulated Brillouin scattering in optical fibers seeded with phase-modulated light," Opt. Exp. 20(19) 21196-21213 (2012) 